\title{
ON CONTINUOUS MULTI-UTILITY REPRESENTATIONS OF SEMI-CLOSED AND CLOSED PREORDERS
}

\author{
Gianni Bosi ${ }^{a}$, Gerhard Herden ${ }^{\mathrm{b}, *}$ \\ ${ }^{a} D E A M S$, Università di Trieste, \\ Piazzale Europa 1, 34127, Trieste, Italy \\ ${ }^{\mathrm{b}}$ Fakultät für Mathematik, Mathematikcarrée, Universität Duisburg-Essen, \\ Campus Essen, Thea-Leymann-Straße 9, D-45127 Essen, Germany
}

\begin{abstract}
On basis of the meanwhile classical continuous multi-utility representation theorem of Levin on locally compact and $\sigma$-compact Hausdorff-spaces the question of characterizing all topological spaces $(X, t)$ for which every semi-closed and closed preorder respectively admits a continuous multi-utility representation will be discussed. This discussion, in particular, provides the fundaments of a mainly topological theory that systematically combines topological and order theoretic aspects of the continuous multi-utility representation problem.
\end{abstract}

Key words: Normal preorder, strongly normal preorder, paracompact space, Lindelöf space, metrizable space

Mathematics subject classification (2010): 54F05, 91B16, 06A05

Proposed running head: Continuous multi-utility representations

\section{Introduction}

A well-known and in some sense best approach (cf. Evren and Ok [6], Bosi and Herden [2] and Alcantud, Bosi and Zuanon [1]) of representing a preorder $\precsim$ (reflexive and transitive relation) on a topological space $(X, t)$ is to find a family $\mathcal{F}$ of continuous real-valued functions $f$ on $(X, t)$ such that

* Corresponding author. Tel.: +49-201-183-2516, Fax: +49-201-183-2426. E-mail address: gerhard.herden@uni-due.de 
" $x \precsim y \Leftrightarrow f(x) \leq f(y)$ " for all $f \in \mathcal{F}$. This equivalence, clearly, implies that every function $f \in \mathcal{F}$ is increasing. Such a representation of $\precsim$ is called a continuous multi-utility representation of $\precsim$. It has the advantage of fully characterizing the preorder $\precsim$.

A total (complete) preorder on a topological space $(X, t)$ is said to be continuous if $\precsim$ satisfies one of the following conditions the equivalence of which is well known.

C0: For every pair $(x, y) \in \prec$ some open decreasing subset $O$ of $X$ and some open increasing subset $U$ of $X$ can be chosen in such a way that $(x, y) \in(O \times(X \backslash O)) \cap((X \backslash U) \times U)$.

C1: For every point $x \in X$ both sets $d(x):=\{y \in X \mid y \precsim x\}$ and $i(x):=\{z \in X \mid x \precsim z\}$ are closed subsets of $X$.

C2: $\precsim$ is a closed subset of $X \times X$ with respect to the product topology $t \times t$ on $X \times X$ that is induced by $t$.

Let $\Delta_{X}=\{(x, x) \mid x \in X\}$ be the diagonal of $X$ and let, furthermore, $\mathcal{P}(M)$ denote for every set $M$ the power set of $M$. Then we note that the implications "C1 $\Rightarrow \mathbf{C} 0$ " and "C2 $\Rightarrow \mathbf{C} 1$ " also hold if $\precsim$ is allowed to be incomplete. In this case, however, the converse implications, in general, fail to be true. In order to verify that the implication "C0 $\Rightarrow \mathbf{C 1}$ " does not hold for not necessarily total (complete) preorders $\precsim$ let $(X, t):=$ $(\{1,2,3,4\}, \mathcal{P}(\{1,3,4\}) \cup\{\{1,2,3,4\}\})$ and $\precsim:=\Delta_{X} \cup\{(1,3)\}$. Then one easily verifies that $\precsim$ satisfies condition $\mathbf{C 0}$ but has the property that neither $d(\{1\})=\{1\}$ nor $i(\{3\})=\{3\}$ are closed subsets of $(X, t)$. Hence, it follows, in particular, that $\precsim$ does not satisfy condition C1. Throughout the literature, however, the weak condition C0 only seems to be of less importance. Therefore, we do not consider condition $\mathbf{C 0}$ in the remainder of this paper. Conversely, it is well-known that in the arbitrary case condition C1 does not imply condition C2. Nevertheless, in Theorem 3.2 we shall discuss the problem up to which degree condition $\mathbf{C 1}$ is weaker than condition C2. Therefore, we still recall that in the literature a preorder $\precsim$ on $(X, t)$ that satisfies condition C1 is said to be semi-closed while a preorder $\precsim$ on $(X, t)$ that satisfies condition C2 is said to be closed. With help of this notation in the framework of our paper the particular importance of clarifying up to which degree condition C1 is weaker than condition C2 is motivated by the following proposition. In order to prove the proposition we need the following elementary and wellknown lemma the proof of which is based upon some straightforward indirect argument and, therefore, may be omitted for the sake of brevity.

Lemma 1.1 Let $\precsim$ be a preorder on $(X, t)$. Then in order that $\precsim$ admits a continuous multi-utility representation it is necessary and sufficient that for any two points $x \in X$ and $y \in X$ such that not $(y \precsim x)$ there exists a continuous 
increasing real-valued function $f_{x y}$ on $(X, t)$ such that $f_{x y}(x)<f_{x y}(y)$.

Proposition 1.1 Let $\precsim$ be a preorder on $(X, t)$ that admits a continuous multi-utility representation. Then $\precsim$ is a closed preorder on $(X, t)$.

\section{Sketch of proof:}

Let there exist a family $\mathcal{F}$ of continuous real-valued functions $f$ on $(X, t)$ such that $x \precsim y \Leftrightarrow f(x) \leq f(y)$ for all $f \in \mathcal{F}$. Then we arbitrarily choose some pair $(x, y) \in X \times X$ such that $\operatorname{not}(x \precsim y)$. In this case Lemma 1.1 guarantees the existence of some function $f \in \mathcal{F}$ such that $f(y)<f(x)$. Then we assume, in contrast, that for every pair $(K, L)$ of disjoint open real intervals $K$ and $L$ such that $(x, y) \in f^{-1}(K) \times f^{-1}(L)$ there exists at least one pair $(u, v) \in f^{-1}(K) \times f^{-1}(L)$ such that $u \precsim v$ in order to conclude with help of some standard "limit argument" that is based upon the continuity of $f$ that $f(x) \leq f(y)$. This contradiction guarantees the existence of disjoint open real intervals $I$ and $J$ such that $(x, y) \in f^{-1}(I) \times f^{-1}(J)$ and $\operatorname{not}(u \precsim v)$ for all pairs $(u, v) \in f^{-1}(I) \times f^{-1}(J)$.

\section{Alternative proof:}

Let for every $f \in \mathcal{F}$ its associated preorder $\precsim_{f}$ be defined by setting $\precsim_{f}:=$ $\{(x, y) \in X \times X \mid f(x) \leq f(y)\}$. Then the continuity of $f$ implies that $\precsim_{f}$ is a total (complete) semiclosed preorder on $(X, t)$. Hence, $\precsim_{f}$ is a closed preorder on $(X, t)$. It, thus, follows that $\precsim=\bigcap_{f \in \mathcal{F}} \precsim f$ is a closed preorder on $(X, t)$.

In addition, it is beyond any doubt that closed preorders are of particular importance in mathematical economics (cf., for instance, the literature that has been quoted by Evren and Ok [6], Bosi and Herden [2], Minguzzi $[15,16]$ and many others). Indeed, in some standard textbooks on microeconomics (such as Mas-Colell, Whinston and Green [14, page 46]) the definitions of continuity of an (incomplete) preference relation and of a closed preference relation coincide. In addition closed preorders are of particular interest in the fundamental work of Nachbin on topology and order (cf. [17, Chapter 1]). In combination with Proposition 1.1, this observation immediately suggests that the most fundamental question in the theory of continuous multi-utility representations of preorders is the question of precisely characterizing (determining) all topological spaces $(X, t)$ for which all their closed preorders admit a continuous multi-utility representation. Nevertheless semiclosed preorders cannot be ignored in mathematical economics (cf. the recent paper by Nosratabadi [18]). But with help of Proposition 1.1 Theorem 3.2 also solves the problem of characterizing (determining) all topological spaces $(X, t)$ for which all their semi-closed preorders admit a continuous multi-utility representation.

Although meanwhile many papers on the continuous multi-utility representation of (closed) preorders have been published (cf., for instance, the 
literature that has been cited in the more recent papers by Bosi and Herden [2], Bosi and Zuanon [3], Evren [5], Evren and Ok [6], Galaabaatar and Karni [7], Minguzzi [15,16] and Pivato [19]) since the pioneering work of Levin [12] (cf. also Evren and Ok [6, Theorem 1]) with respect to the above mentioned fundamental problem in the theory of continuous multi-utility representations no real progress has been made. Levin's fundamental theorem states that every closed preorder on a locally and $\sigma$-compact Hausdorff space has a continuous multi-utility representation. Indeed, in combination with its corollaries on separable metric spaces, compact spaces and Euclidean spaces (cf., for instance, Evren and Ok [6, Corollary 1, Corollary 2 and Corollary 3]) this theorem still belongs to the most quoted theorems in Mathematical Utility Theory, in particular, in the theory of appropriate utility representations of incomplete preference relations.

To the best of our knowledge all well-known continuous multi-utility representation theorems only present as well as Levin's theorem sufficient conditions for the existence of continuous multi-utility representations. In opinion of the authors this is the great lack of these theorems. This lack pertains to formal mathematics as well as to applications in mathematical economics. Indeed, in order to completely characterizing (determining) topological spaces $(X, t)$ having the property that all their semi-closed and closed preorders respectively admit a continuous multi-utility representation necessary and sufficient conditions have to be presented. In mathematical economics, on the other hand, necessary conditions allow the selection of appropriate topologies. Indeed, necessary conditions imply at least particular difficulties in representing a preorder by a family of continuous increasing real-valued functions. Actually, these conditions even often imply the impossibility of a continuous multi-utility representation of a preorder (cf. the examples that have been presented in the conclusion of this paper).

The difficulties of presenting necessary conditions for the existence of a family of continuous increasing real-valued functions that represent a (closed) preorder is based upon the fact that corresponding proofs must be constructive. Indeed, proving the necessity of a given condition one, in general, is forced to verify that negating the validity of this condition allows the construction of (closed) preorders that do not have a continuous multi-utility representation. Corresponding proofs, therefore, need the intuitive idea of possible conditions that may be necessary for representing a preorder by a family of continuous real-valued functions as well as the ability of constructing preorders that do not have a continuous multi-utility representation if these conditions are not satisfied.

Because of these difficulties our approach of approximating the problem of completely characterizing topological spaces $(X, t)$ for which every closed respectively, semi-closed preorder admits a continuous multi-utility representation, therefore, is conservative. This means that Levin's original theorem stands in focus of our approach. In a first attempt we, therefore, want to clarify up to which degree the assumptions of locally and $\sigma$-compactness are also 
necessary for ensuring the existence of continuous multi-utility representations for closed preorders. Indeed, setting $S:=\{x \in X \mid\{x\} \in t\}$ and concentrating on metrizable spaces in this way in the third section of this paper, among other results, the following three results will be proved and widely generalized (cf. Theorem 3.5, Theorem 3.7, Theorem 3.8 and corresponding corollaries).

\section{First result on metrizable spaces:}

Let $(X, t)$ be a metrizable space. Then one of the following assertions holds:

(i) $\left(X \backslash S, t_{\mid X \backslash S}\right)$ is compact. In this case every closed preorder $\precsim$ on $(X, t)$ admits a continuous multi-utility representation.

(ii) $\left(X \backslash S, t_{\mid X \backslash S}\right)$ is not compact. Then in order that every closed preorder $\precsim$ on $(X, t)$ admits a continuous multi-utility representation it is necessary that $(X, t)$ is the direct sum of locally compact second countable metric spaces.

\section{Second result on metrizable spaces:}

Let $(X, t)$ be a second countable space. Then in order that every closed preorder $\precsim$ on $(X, t)$ admits a continuous multi-utility representation it is necessary and sufficient that $\left(X \backslash S, t_{\mid X \backslash S}\right)$ is a compact and $(X, t)$ a second countable metrizable space or that $(X, t)$ is a second countable locally compact metrizable space.

\section{Third result on metrizable spaces:}

Let $(X, t)$ be a connected metrizable space. Then in order that every closed preorder $\precsim$ on $(X, t)$ admits a continuous multi-utility representation it is necessary and sufficient that $(X, t)$ is locally compact and second countable.

In addition, Theorem 3.2 (cf. Remark 3.1) states that for a first countable space $(X, t)$ the assertions $(X, t)$ to have the property that every semi-closed preorder is closed, $(X, t)$ to have the property that every semi-closed preorder admits a continuous multi-utility representation and $(X, t)$ to contain at most one point $x \in X$ such that $\{x\}$ is not an open subset of $X$ are equivalent. In this paper topological spaces $(X, t)$ for which there exists at most one point $x \in X$ such that $\{x\}$ is not an open subset of $X$ are said to be almost discrete. Furthermore, the negation of the existence of weakly inaccessible cardinal numbers allows us to drop the assumption $(X, t)$ to be first countable in order to nevertheless prove a corresponding very general restrictive result (cf. assertion (ii) of Theorem 3.2). This result combines our approach with the theory of large cardinal numbers. 


\section{Additional notation and preliminaries}

As usual $t_{\text {nat }}$ denotes the natural topology on the real line. $|M|$ denotes for every set $M$ the cardinality of $M$.

Let, in the remainder of this paper, $\precsim$ be a preorder on some fixed given set $X$. Then a subset $D$ of $X$ is said to be decreasing if $d(x) \subset D$ for all $x \in D$. By duality the concept of an increasing subset $I$ of $X$ is defined.

In addition, for every subset $T$ of $X$ we set

$d(T):=\{y \in X \mid \exists x \in T(y \precsim x)\}$,

$i(T):=\{z \in X \mid \exists x \in T(x \precsim z)\}$.

Therefore, $d(T)$ is the smallest decreasing and $i(T)$ the smallest increasing subset of $X$ that contains $T$.

Let $t$ be a topology on $X$. As usual we denote for every subset $S$ of $X$ by $\bar{S}$ its topological closure. For every subset $T$ of $X$ we denote, furthermore, by $D(T)$ the smallest closed decreasing subset of $X$ that contains $T$. Analogously, we denote by $I(T)$ the smallest closed increasing subset of $X$ that contains $T$.

Furthermore, $\precsim$ is said to be

(i) $d$-i-closed if for every closed subset $A$ of $X$ both sets $d(A)$ and $i(A)$ are closed subsets of $\mathrm{X}$. (The reader may recall that $(X, t)$ is a Fréchet-space if and only if $\{x\}$ is a closed subset of $X$ for every point $x \in X$. Therefore, in a Fréchet-space $(X, t)$ every $d$ - $i$-closed preorder is semi-closed.).

(ii) $D$-I-closed if for any two closed subsets $A$ and $B$ of $X$ such that $\operatorname{not}(y \precsim x)$ for all $x \in A$ and all $y \in B$ the sets $D(A)$ and $I(B)$ are disjoint. (Since the relation $\operatorname{not}(y \precsim x$ ) for all $x \in A$ and all $y \in B$ means that $d(A) \cap i(B)=\emptyset$ it follows that a $d$-i-closed preorder $\precsim$ on $(X, t)$ also is $D$ $I$-closed. In case that $(X, t)$ is a regular space also the converse implication holds (cf. Proposition 3.1)).

(iii) normal if for any two disjoint closed decreasing, respectively increasing subsets $A$ and $B$ of $X$ there exist disjoint open decreasing, respectively increasing subsets $U$ and $V$ of $X$ such that $A \subset U$ and $B \subset V$.

(iv) strongly normal if for any two closed subsets $A$ and $B$ of $X$ such that $\operatorname{not}(y \precsim x)$ for all $x \in A$ and all $y \in B$ there exist disjoint open decreasing, respectively increasing subsets $U$ and $V$ of $X$ such that $A \subset U$ and $B \subset V$.

Finally, $\precsim$ is said to satisfy cmp (continuous multi-utility representation property) if $\precsim$ admits a continuous multi-utility representation. 


\section{$3 \quad$ The results}

Let throughout this section $(X, t)$ be an arbitrarily chosen Fréchet-space. The assumption $(X, t)$ to be a Fréchet-space does not mean any loss of generality. In order to realize this observation let the topological space $(X, t)$ be arbitrarily chosen. Then we consider the equivalence relation " $\approx$ " on $X$ that for all points $x \in X$ and $y \in X$ is defined by setting " $x \approx y \Leftrightarrow \overline{\{x\}}=\overline{\{y\}}$ " in order to then replace $(X, t)$ by the quotient space $\left(X_{\mid \approx}, t_{\mid \approx}\right)$. Obviously $\left(X_{\mid \approx}, t_{\mid \approx}\right)$ is a Fréchet-space. In addition, for every point $x \in X$ the closure properties of $\overline{\{x\}}$ imply that for every open subset $O$ of $X$ and every closed subset $C$ of $X$ respectively the equality $O=\bigcup_{x \in O} \overline{\{x\}}$ and $C=\bigcup_{x \in C} \overline{\{x\}}$ respectively holds. Hence, the canonical projection $p:(X, t) \rightarrow\left(X_{\mid \approx}, t_{\mid \approx}\right)$ is open and closed. If we now choose an arbitrary preorder $\precsim$ on $(X, t)$ then we may conclude, in particular, that a subset $V$ of $X$ is open and decreasing (open and increasing) or closed and decreasing (closed and increasing) with respect to $\precsim$ if and only if $p(V)$ is with respect to $\precsim \mid \approx$ an open and decreasing (open and increasing) or closed and decreasing (closed and increasing) subset of $\left(X_{\mid \approx}, \tau \mid \approx\right)$. Hence, our concentration on Fréchet-spaces, actually, means no loss of generality.

Now we continue our considerations by at first proving the validity of the following results that at least approximate our aims that have been presented in the introduction. In order to state our first result we need the following two lemmas.

Lemma 3.1 Let $(X, t)$ be a normal space. Then every $d$-i-closed as well as every $D$-I-closed preorder $\precsim$ on $(X, t)$ is strongly normal.

Proof. Since $d(C) \subset D(C)$ and $i(C) \subset I(C)$ for every closed subset $C$ of $X$ it suffices to verify that every $D$ - $I$-closed preorder $\precsim$ on $(X, t)$ is strongly normal. Let, therefore, $A$ and $B$ be two closed subsets of $X$ such that not $(y \precsim x$ ) for all $x \in A$ and all $y \in B$. Then the assumption $D(A)$ and $I(B)$ to be disjoint subsets of $X$ implies with help of the normality of $(X, t)$ that there exists some open subset $O$ of $X$ such that the inclusions $D(A) \subset O \subset \bar{O} \subset X \backslash I(B)$ hold. Since $X \backslash I(B)$ is decreasing it, thus, follows that $d(\bar{O}) \subset X \backslash I(B)$. This inclusion allows us to conclude that not $(y \precsim x)$ for all $x \in \bar{O}$ and all $y \in i(B)$. Hence our assumption $\precsim$ to be $D$-I-closed implies that $D(\bar{O}) \cap I(B)=\emptyset$. Therefore, we set $V:=X \backslash D(\bar{O})$ in order to conclude that $V$ is an open decreasing subset of $X$ that contains $I(B)$. We proceed by considering the inclusions $V=X \backslash D(\bar{O}) \subset X \backslash \bar{O} \subset X \backslash O \subset X \backslash D(A)$. These inclusions imply, in particular, that $\bar{V} \subset X \backslash O \subset X \backslash D(A)$. Since $X \backslash D(A)$ is increasing, we, thus, may conclude that $D(A) \cap i(\bar{V})=\emptyset$, which means that not $(y \precsim x$ ) for all $x \in D(A)$ and all $y \in \bar{V}$. In the same way as above it, therefore, follows that $D(A) \cap I(\bar{V})=\emptyset$. Hence, we set $U:=X \backslash I(\bar{V})$. Then $U$ is an 
open decreasing subset of $X$ that contains $D(A)$. In addition, the inclusion $V \subset I(\bar{V})$ implies that $U \cap V=\emptyset$. Since $A \subset D(A)$ and $B \subset I(B)$ this equality completes the proof of the lemma.

The next lemma already has been proved in Herden [10, Theorem 2.2].

Lemma 3.2 Let $(X, \preceq, t)$ be a preordered topological space. Then the following assertions are equivalent:

(i) $\precsim$ is strongly normal.

(ii) For any two closed subsets $A$ and $B$ of $X$ such that not $(y \precsim x)$ for all $x \in A$ and all $y \in B$ there exists a continuous and increasing function $f_{A B}:(X, \precsim, t) \longrightarrow\left([0,1], \leq, t_{n a t}\right)$ such that $f_{A B}(A)=\{0\}$ and $f_{A B}(B)=\{1\}$.

(iii) For every closed subset $C$ of $X$ and every bounded, continuous and increasing function $f_{C}:\left(C, \precsim_{\mid C}, t_{\mid C}\right) \longrightarrow\left(\mathbb{R}, \leq, t_{\text {nat }}\right)$ there exists a bounded continuous and increasing function $f:(X, \precsim, t) \longrightarrow\left(\mathbb{R}, \leq, t_{\text {nat }}\right)$ such that $f_{\mid C}=f_{C}$.

With help of these lemmas we now are fully prepared for proving the following theorem (cf. Theorem 4.3 and Theorem 4.5 in Bosi and Herden [2]).

Theorem 3.1 The following assertions are equivalent:

(i) Every d-i-closed preorder $\precsim$ on $(X, t)$ satisfies cmp.

(ii) Every $D$-I-closed preorder $\precsim$ on $(X, t)$ satisfies cmp.

(iii) $(X, t)$ is a normal space.

\section{Proof:}

(ii) $\Rightarrow$ (i): With help of the corresponding definitions it follows that every $d$-i-closed preorder $\precsim$ on $X, t)$ is $D$ - $I$-closed (cf. the corresponding part in the proof of Lemma 3.1). Hence, nothing has to be shown.

(i) $\Rightarrow$ (iii): Let $A$ and $B$ be two disjoint closed subsets of $X$. Then we consider the preorder $\precsim$ on $(X, t)$ that is defined by setting

$\precsim:=\Delta_{X} \cup A \times A \cup B \times B$. 
For every closed subset $C$ of $X$ we then may conclude that

$$
d(C)=i(C)=\left\{\begin{array}{ll}
A \cup B \cup C & \text { if } A \cap C \neq \emptyset \text { and } B \cap C \neq \emptyset \\
A \cup C & \text { if } A \cap C \neq \emptyset \text { and } B \cap C=\emptyset \\
B \cup C & \text { if } A \cap C=\emptyset \text { and } B \cap C \neq \emptyset \\
C & \text { if } A \cap C=\emptyset \text { and } B \cap C=\emptyset
\end{array} .\right.
$$

Hence, $\precsim$ is a $d$-i-closed preorder on $(X, t)$. Let, therefore, $x \in A$ and $y \in B$ be arbitrarily chosen. Then the relation $\operatorname{not}(y \precsim x)$ is satisfied. Lemma 1.1, thus, implies the existence of some continuous and increasing real-valued function $f_{x y}$ on $(X, t)$ such that $f_{x y}(x)<f_{x y}(y)$. Since $x \sim u$ for every $u \in A$ and $y \sim v$ for every $v \in B$ it follows that $(X, t)$ is a normal space.

(iii) $\Rightarrow$ (ii): Let $\precsim$ be some $D$ - $I$-closed preorder on $(X, t)$. Then Lemma 3.1 implies that $\precsim$ is strongly normal. Hence, we may apply assertion (ii) of Lemma 3.2 in order to conclude with help of Lemma 1.1 that $\precsim$ satisfies cmp.

We now come to Proposition 3.1 that already has been announced in the previous section.

Proposition 3.1 Let $(X, t)$ be a regular space. Then every D-I-closed preorder $\precsim$ on $(X, t)$ is d-i-closed.

Proof: Let $A$ be a closed subset of $X$. Then we must show that $d(A)$ as well as $i(A)$ are closed subsets of $X$. Of course, it suffices to verify that $d(A)$ is closed. A dual argument then also applies for $i(A)$. Let, therefore, some point $x \in X \backslash d(A)$ be arbitrarily chosen. Then $\operatorname{not}(x \precsim y)$ for all points $y \in A$. Hence, $D(A) \cap I(\{x\})=\emptyset$. The regularity of $(X, t)$ now implies the existence of disjoint open subsets $U$ and $V$ of $X$ such that $D(A) \subset U$ and $x \in V$. Since $d(A) \subset D(A)$ we are done.

The following proposition completes Theorem 3.1.

Proposition 3.2 The following assertions are equivalent:

(i) Every normal preorder $\precsim$ on $(X, t)$ is strongly normal.

(ii) Every normal preorder $\precsim$ on $(X, t)$ is $D$-I-closed.

\section{Proof:}

(i) $\Rightarrow$ (ii): Let $A$ and $B$ be two closed subsets of $X$ such that not $(y \precsim x)$ for all $x \in A$ and all $y \in B$. Then assertion (i) allows us to apply assertion (ii) of Lemma 3.2. This means, in particular, that the sets $D(A)$ and $I(B)$ are 
disjoint.

(ii) $\Rightarrow$ (i): Let $\precsim$ be normal and let $A$ and $B$ be closed subsets of $X$ such that $\operatorname{not}(y \precsim x)$ for all $x \in A$ and all $y \in B$. Then assertion (ii) implies that $D(A)$ and $I(B)$ are disjoint closed decreasing, respectively increasing subsets of $X$. Since $\precsim$ is normal it, thus, follows that there exist disjoint open decreasing, respectively increasing subsets $U$ and $V$ of $X$ such that $D(A) \subset U$ and $I(B) \subset V$. The inclusions $A \subset D(A)$ and $B \subset I(B)$ now imply the validity of assertion (i).

Let us concentrate, for the moment, on Hausdorff spaces $(X, t)$ in order to then at first discuss the problem of characterizing all Hausdorff spaces $(X, t)$ having the property that every semi-closed preorder $\precsim$ on $(X, t)$ is closed and the problem of characterizing all Hausdorff spaces $(X, t)$ having the property that every semi-closed preorder $\precsim$ on $(X, t)$ satisfies cmp. In order to start this discussion the reader may recall from the introduction that $S:=\{x \in X \mid\{x\} \in t\}$. In addition, the following notation will be used.

N1: By $D$ we abbreviate the set of all points $x \in X$ that have the property that every neighborhood of $x$ contains some point $y \in X \backslash S$.

N2: For every point $x \in X$ we set

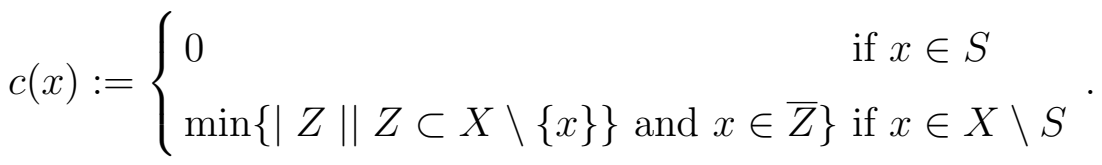

For every point $x \in X \backslash S$ it follows that $c(x) \geq \aleph_{0}$ and that $c(x)$ is a regular cardinal number. Indeed, $c(x)$ is the smallest cardinal number $\kappa$ for which there exists a basis $\mathcal{U}(x)$ of (open) neighborhoods of $x$ such that $|\mathcal{U}(x)|=\kappa$. Let us abbreviate these observations by $\left(^{*}\right)$.

N3: By $Z F C+\neg W I$ we abbreviate the extension of Zermelo-Fraenkel set theory + Axiom of Choice that negates the existence of weakly inaccessible cardinal numbers, i.e. the existence of uncountable regular cardinal numbers $\kappa$ having the property that for every cardinal number $\gamma$ that is strictly smaller than $\kappa$ also its successor is strictly smaller than $\kappa$. It is well known that the consistency of $Z F C$ implies the consistency of $Z F C+\neg W I$.

With help of this notation we are fully prepared for stating the following theorem.

Theorem 3.2 The following assertions hold in $Z F C$ and $Z F C+\neg W I$ respectively:

(i) In ZFC it can be proved that in order that every semi-closed preorder 
on $(X, t)$ is closed it is necessary and sufficient that $c(x) \neq c(y)$ for any two different points $x \in X \backslash S$ and $y \in X \backslash S$.

(ii) In $Z F C+\neg W I$ the following assertions are equivalent:

SC: Every semi-closed preorder $\precsim$ on $(X, t)$ is closed.

SM: Every semi-closed preorder $\precsim$ on $(X, t)$ satisfies cmp.

ST: $(X, t)$ satisfies the following conditions:

ST1: $c(x) \neq c(y)$ for any two different points $x \in X \backslash S$ and $y \in X \backslash S$.

ST2: $X=\bar{S}$.

ST3: $t_{\mid X \backslash S}$ is the discrete topology on $X \backslash S$.

\section{Proof:}

(i): Necessity: Let us assume, in contrast, that there exist at least two different points $x \in X \backslash S$ and $y \in X \backslash S$ such that $c(x)=c(y)$. Then we choose subsets $Z$ of $X \backslash\{x\}$ and $Z^{\prime}$ of $X \backslash\{y\}$ in such a way that $|Z|=c(x)=$ $c(y)=\left|Z^{\prime}\right|$ and $x \in \bar{Z}$ and $y \in \overline{Z^{\prime}}$. Since $(X, t)$ is assumed to be a Hausdorff space we may assume without loss of generality that $Z \cap Z^{\prime}=\emptyset$. Hence, we may consider some bijective function $\phi: Z \longrightarrow Z^{\prime}$ in order to choose the (pre)order $\preceq$ on $X$ that is defined by setting

$\preceq:=\Delta_{X} \cup\{(z, \phi(z)) \mid z \in Z\}$.

Since for every $z \in Z$ the singletons $\{z\}$ and $\{\phi(z)\}$ are closed subsets of $X$ it follows that $\preceq$ is a semi-closed (pre)order on $(X, t)$. The assumption of assertion (i), thus, implies that $\preceq$ is a closed (pre)order on $(X, t)$. Since $(X, t)$ is a Hausdorff space it, thus, follows that $(x, y) \in \precsim$. But since $(x, y) \notin \precsim$ this conclusion is incompatible with the definition of $\preceq$. This contradiction proves the necessity part of assertion (i).

Sufficiency: Let us assume, in contrast, that there exists some semi-closed preorder $\precsim$ on $(X, t)$ that is not closed. Then there exists some cardinal number $\kappa$ and a set $\left\{\left(x_{\alpha}, y_{\alpha}\right) \mid \alpha<\kappa\right\}$ of pairs $\left(x_{\alpha}, y_{\alpha}\right) \in \precsim$ that is not closed with respect to the product topology $t \times t$ on $X \times X$. This means that we may assume without loss of generality that there exists some pair $(x, y) \in X \times(X \backslash\{x\})$ such that $(x, y) \in \overline{\left\{\left(x_{\alpha}, y_{\alpha}\right) \mid \alpha<\kappa\right\}}$ but $(x, y) \notin \precsim$. Of course, we may assume, in addition, that there exists no cardinal number $\lambda<\kappa$ such that $(x, y) \in \overline{\left\{\left(x_{\alpha}, y_{\alpha}\right) \mid \alpha<\lambda\right\}}$. It, thus, follows that $c(x)=c(y)=\kappa$. Since $x \neq y$ this conclusion contradicts the assumption of assertion (ii) and, therefore, finishes the proof of the sufficiency part of assertion (ii).

(ii): We now assume the validity of $Z F C+\neg W I$. Since a preorder $\precsim$ on $(X, t)$ that admits a continuous multi-utility representation is closed the proof of the validity of the implication "SM $\Rightarrow$ SC" does not need any additional reflection. We, thus, only have to prove that the implications "SC $\Rightarrow$ ST" and "ST $\Rightarrow$ SM" hold. In order to show the validity of the 
implication "SC $\Rightarrow \mathbf{S T}$ " let every semi-closed preorder $\precsim$ on $(X, t)$ be closed. Because of assertion (i) it suffices to verify the validity of the conditions ST2 and ST3. The proof of these conditions is divided into two steps.

In the first step we arbitrarily choose some point $x \in D$ in order to then show that $|U| \geq \min \{c(z) \mid z \in X$ and $c(x)<c(z)\}$ for every neighborhood $U$ of $x$. Because of observation $\left(^{*}\right)$ the desired inequality follows for all neighborhoods $U$ of $x$ if we are able to prove that every neighborhood $U$ of $x$ contains at least one point $y$ such that $c(x)<c(y)$. Let us assume, in contrast, that $c(y) \leq c(x)$ for every neighborhood $U$ of $x$ and every point $y \in U \backslash\{x\}$. Then assertion (i) implies that $c(y)<c(x)$ for every neighborhood $U$ of $x$ and every point $y \in U \backslash\{x\}$. Hence, the regularity of $c(x)$ implies with help of assertion (i) that $\sup c(y)=c(x)$. This equation allows us to conclude that for every cardinal number $\gamma$ that is strictly smaller than $c(x)$ there exists some cardinal number $\lambda$ that is strictly greater than $\gamma$ and strictly smaller than $c(x)$. The regularity of $c(x)$, therefore, implies that $c(x)$ is a weakly inaccessible cardinal number which contradicts our assumption that there exist no weakly inaccessible cardinal numbers and, thus, finishes the proof of the first step.

In the second step we, finally, show that $D$ is empty. Then both conditions ST2 and ST3 have been proved.. Let us assume, in contrast, that $D$ is not empty. Then there exists some point $x \in D$ such that $c(x)=\min _{w \in T} c(w)$. In addition, the proof of the first step implies the existence of some point $y \in X$ such that $c(y)=\min \{c(v) \mid v \in X$ and $c(x)<c(v)\}$. Now the first step allows us to recursively construct a collection $\left\{Z_{\alpha}\right\}_{\alpha<c(y)}$ of pairwise disjoint subsets $Z_{\alpha}$ of $X \backslash\{x\}$ for which the equation $\left|Z_{\alpha}\right|=c(x)$ and the inclusion $x \in \overline{Z_{\alpha}}$ hold. Since the sets $Z_{\alpha}$ are pairwise disjoint it follows that $\left|\bigcup_{\alpha<c(y)} Z_{\alpha}\right|=c(y)$. We, thus, set $Z:=\bigcup_{\alpha<c(y)} Z_{\alpha}$ and consider, in addition, some subset $Z^{\prime}$ of $X \backslash\{y\}$ in such a way that $\left|Z^{\prime}\right|=c(y)$ and $y \in \overline{Z^{\prime}}$. As in the proof of assertion (i) we proceed by choosing some bijective map $\phi: Z \longrightarrow Z^{\prime}$ in order to then considering as in the proof of assertion (i) the semi-closed preorder

$\preceq:=\Delta_{X} \cup\{(z, \phi(z)) \mid z \in Z\}$

on $(X, t)$. Now we may conclude as in the proof of assertion (i). This means that the assumption $\precsim$ to be a closed preorder on $(X, t)$ implies that $(x, y) \in \precsim$ in contrast to the definition of $\precsim$ which finishes the proof of the implication "SC $\Rightarrow$ ST".

In order to now finally prove the validity of the implication "ST $\Rightarrow \mathbf{S M}$ " we first apply Lemma 1.1 and assertion (i) of the theorem in order to conclude with help of condition ST1 that it suffices to verify that every closed preorder $\precsim$ on $(X, t)$ is strongly normally preordered. Let, therefore, $\precsim$ be some closed preorder on $(X, t)$. Then we arbitrarily choose closed subsets $A$ and $B$ of $X$ 
such that $\operatorname{not}(y \precsim x)$ for any two points $x \in A$ and $y \in B$. We must show that there exist disjoint open decreasing respectively, increasing subsets $U$ and $V$ of $X$ such that $A \subset U$ and $B \subset V$. $U$ and $V$ will be constructed inductively.

$n=0$ : Indeed, the validity of the conditions ST2 and ST3 implies the existence of disjoint open subsets $O_{0}$ and $P_{0}$ of $X$ such that $A \subset O_{0}$ and $B \subset P_{0}$. Unfortunately, it cannot be excluded that there exist pairs $(x, y) \in$ $\left(O_{0}, P_{0}\right)$ such that $y \precsim x$. But, whatever the case may be, we choose the sets $K:=\left\{y \in P_{0} \mid \exists x \in A(y \precsim x)\right\}, L:=\left\{x \in O_{0} \mid \exists y \in B(y \precsim x)\right\}$ and $M:=\left\{y \in P_{0} \backslash K \mid \exists x \in O_{0}(y \precsim x)\right\}$. Then the assumption $\precsim$ to be closed implies that the sets $L$ and $K \cup M$ are closed subsets of $O_{0} \backslash A$ and $P_{0} \backslash B$ respectively. Therefore, we set $O_{0}^{\prime}:=O_{0} \backslash L$ and $P_{0}^{\prime}:=P_{0} \backslash(K \cup M)$. Then we may conclude that the open subsets $O_{0}^{\prime}$ and $P_{0}^{\prime}$ of $X$ have the properties that $A \subset O_{0}^{\prime}$ and $B \subset P_{0}^{\prime}$ and, furthermore, that $d\left(\overline{d\left(O_{0}^{\prime}\right)}\right) \cap i\left(\overline{i\left(P_{0}^{\prime}\right)}\right)=\emptyset$.

$0 \Rightarrow 1$ : Now we assume that already open subsets $O_{0}^{\prime}$ and $P_{0}^{\prime}$ of $X$ have been constructed in such a way that $A \subset O_{n}^{\prime}, B \subset P_{n}^{\prime}$ and $d\left(\overline{d\left(O_{0}^{\prime}\right)}\right) \cap i\left(\overline{i\left(P_{0}^{\prime}\right)}\right)=$ $\emptyset$. Then the same argument that has been applied for $n=0$ allows us to construct open subsets $O_{1}^{\prime}$ and $P_{1}^{\prime}$ of $X$ such that $\overline{d\left(O_{0}^{\prime}\right)} \subset O_{1}^{\prime}$ and $\overline{i\left(P_{0}^{\prime}\right)} \subset P_{1}^{\prime}$ and $d\left(\overline{d\left(O_{1}^{\prime}\right)}\right) \cap i\left(\overline{i\left(P_{1}^{\prime}\right)}\right)=\emptyset$.

$0<n \Rightarrow n+1$ : In this situation we assume that open subsets $O_{n}^{\prime}$ and $P_{n}^{\prime}$ of $X$ have been constructed in such a way that the inclusions $\overline{d\left(O_{n-1}^{\prime}\right)} \subset O_{n}^{\prime}$ and $\overline{i\left(P_{n-1}^{\prime}\right)} \subset P_{n}^{\prime}$ and the equation $d\left(\overline{d\left(O_{0}^{\prime}\right)}\right) \cap i\left(\overline{i\left(P_{0}^{\prime}\right)}\right)=\emptyset$ are satisfied. Then the same argument that has been applied for $n=0$ allows us to construct open subsets $O_{n+1}^{\prime}$ and $P_{n+1}^{\prime}$ of $X$ such that $\overline{d\left(O_{n}^{\prime}\right)} \subset O_{n+1}^{\prime}$ and $\overline{i\left(P_{n}^{\prime}\right)} \subset P_{n+1}^{\prime}$ and $d\left(\overline{d\left(O_{n+1}^{\prime}\right)}\right) \cap i\left(\overline{i\left(P_{n+1}^{\prime}\right)}\right)=\emptyset$.

Continuing in this way we, finally, set $U:=\bigcup_{n=0}^{\infty} O_{n}^{\prime}$ and $V:=\bigcup_{n=0}^{\infty} P_{n}^{\prime}$. The inductive construction of the open subsets $O_{n}^{\prime}$ and $P_{n}^{\prime}$ of $X$ implies that $U$ and $V$ are disjoint open decreasing respectively, increasing subsets of $X$ such that $A \subset U$ and $B \subset V$. This conclusion finishes the proof of the implication "ST $\Rightarrow$ SM" and, thus, completes the proof of the theorem.

Remark 3.1 The reader may notice that the proof of the implication "ST $\Rightarrow \mathbf{S M " ~ d o e s ~ n o t ~ m a k e ~ a n y ~ u s e ~ o f ~} \neg W I$. This implication, therefore, also holds in $Z F C$. In addition, the proof of the implication "ST $\Rightarrow \mathbf{S M}$ " allows us to conclude that for a topological space $(X, t)$ that satisfies the conditions ST2 and ST3 every closed preorder $\precsim$ on $(X, t)$ is strongly normal. In Proposition 3.5 a somewhat weaker result will be proved for Hausdorff-spaces $(X, t)$ having the property that $\left(X \backslash S, t_{X \backslash S}\right)$ is compact.

Assertion (i) of Theorem 3.2 implies that a Hausdorff space $(X, t)$ that contains at most one point $x$ such that $\{x\} \in t$ and has the property that every semi-closed preorder $\precsim$ on $(X, t)$ already is closed must be rigid. This means that the only one homeomorphism $\phi:(X, t) \longrightarrow(X, t)$ is the identity map on $X$.

Assertion (ii) of Theorem 3.2 implies that the assertion that there exists 
no connected Hausdorff space $(X, t)$ for which every semi-closed preorder $\precsim$ on $(X, t)$ already is closed is consistent with $Z F C$.

Let $\kappa$ be an infinite regular cardinal number. Then a topological space $(X, t)$ is said to be $\kappa$-countable if every point $x \in X$ either is contained in $S$ or has a basis of neighborhoods the cardinality of which is $\kappa$. If $\kappa=\aleph_{0}$ then $(X, t)$ is first countable. Now the reader may still recall from the introduction that a topological space $(X, t)$ is said to be almost discrete if $|X \backslash S| \leq 1$. Let $(X, t)$ be a $\kappa$-countable Hausdorff space. Then assertion (i) of Theorem 3.2 immediately implies that in $Z F C$ the validity of the equivalence of the following assertions holds.

(i) Every semi-closed preorder $\precsim$ on $(X, t)$ is closed.

(ii) Every semi-closed preorder $\precsim$ on $(X, t)$ satisfies cmp.

(iii) $(X, t)$ is almost discrete.

Finally, we want to complete the above considerations on semi-closed, closed, $d$ - $i$-closed and $D$ - $I$-closed preorders by proving the validity of the following proposition.

Proposition 3.3 Let $(X, t)$ contain at least one point that has a countable and infinite basis of neighborhoods. Then the following assertions hold:

(i) In order that every closed preorder $\precsim$ on $(X, t)$ is $d$-i-closed it is necessary that $(X, t)$ is sequentially compact.

(ii) In order that every closed preorder $\precsim$ on $(X, t)$ is $D$-I-closed it is necessary that $(X, t)$ is sequentially compact.

Proof: We shall prove both assertions of the proposition in one step. Therefore, we assume, in contrast, that there exists some countable infinite subset $C$ of $X$ that does not have a limit point in order to then use our assumption on $(X, t)$ for choosing a sequence $\left(x_{n}\right)_{n \in \mathbb{N}}$ of points $x_{n} \in X$ that converges to some point $x \in X$. Let $D:=\left\{x_{n} \mid n \in \mathbb{N}\right\}$. Of course, we may assume that $x \notin D$. We, thus, proceed by dividing $C$ into two disjoint infinite subsets $A$ and $B$ and $D$ into two disjoint infinite subsets $H$ and $K$ the union of which is $C$ and $D$ respectively. Now we consider bijective functions $\phi: H \longrightarrow A$ and $\psi: B \longrightarrow K$. With help of these functions we may define a (pre)order $\precsim$ on $(X, t)$ by setting

$\precsim:=\Delta_{X} \cup\{(h, \phi(h)) \mid h \in H\} \cup\{(b, \psi(b)) \mid b \in B\}$. 
Since both sets $A$ and $B$ are closed subsets of $X$ and $(X, t)$ is a Hausdorff space it follows that $\precsim$, actually, is a closed (pre)order on $(X, t)$. But since $x$ neither is contained in $d(A)$ nor in $i(B)$ we may conclude that $\precsim$ is not $d$-i-closed. In addition, the relation $x \in \overline{d(A)} \cap \overline{i(B)}$ implies that $\precsim$ is not $D$-I-closed. These contradictions prove the proposition.

The following theorem is well known in general topology (cf., for instance, Grotemeyer [8, Satz 93]). The reader may notice that we now drop the assumption $(X, t)$ to be a Hausdorff space.

Theorem 3.3 Let $(X, t)$ be paracompact. Then the following assertions are equivalent:

(i) $(X, t)$ is compact.

(ii) $(X, t)$ is sequentially compact.

As an application of Theorem 3.3 we want to apply Proposition 3.3 in order to prove the following proposition.

Proposition 3.4 Let $(X, t)$ be a paracompact space that contains at least one point that has a countable and infinite basis of neighborhoods. Then the following assertions are equivalent:

(i) Every closed preorder $\precsim$ on $(X, t)$ is d-i-closed..

(ii) Every closed preorder $\precsim$ on $(X, t)$ is $D$-I-closed..

(iii) $(X, t)$ is a compact Hausdorff space.

\section{Proof:}

(i) $\Rightarrow$ (ii): The validity of this implication is trivial (cf. the corresponding remark in the proof of Lemma 3.1).

(ii) $\Rightarrow$ (iii): Since a paracompact space is normal our assumption $(X, t)$ to be a Fréchet space implies that $(X, t)$ is a Hausdorff space. Hence, the validity of the implication "(ii) $\Rightarrow$ (iii)" is a consequence of Proposition 3.3 and Theorem 3.3.

(iii) $\Rightarrow$ (i): The validity of this implication is well known. The reader may consult, for instance, the proof of Proposition 4 in Nachbin [17, Chapter 3]. $\square$

In the remainder of this paper we now solely concentrate on the problem of characterizing all topological spaces $(X, t)$ that have the property that all their closed preorders $\precsim$ satisfy cmp. In order to at least approach this problem the following general theorem seems to be of interest (cf. Proposition 3.4).

Theorem 3.4 Let $(X, t)$ be a Hausdorff space. Then the following assertions are equivalent: 
(i) Every closed preorder $\precsim$ on $(X, t)$ satisfies cmp..

(ii) Every closed preorder $\precsim$ on $(X, t)$ is normal.

Proof: $(\mathrm{i}) \Rightarrow$ (ii): Let $\precsim$ be a closed preorder on $(X, t)$ and let disjoint closed subsets $A$ and $B$ of $X$ be chosen in such a way that $A$ is decreasing and $B$ is increasing with respect to $\precsim$. Then we at first set $\precsim e:=\precsim \cup A \times A \cup B \times B$. Since $(X, t)$ is a Hausdorff space it follows that $\precsim e$ is a closed binary relation on $(X, t)$. In order to now construct the transitive closure $\precsim^{t}$ of $\precsim^{e}$ we set $A_{r}:=\{u \in X \mid \exists a \in A(a \precsim u)\}$ and $B_{l}:=\{v \in X \mid \exists b \in B(v \precsim b)\}$. Because of our assumptions $A$ to be decreasing and $B$ to be increasing we may conclude that $\precsim^{t}=\precsim^{e} \cup A \times A_{r} \cup B_{l} \times B$. Since $\precsim$ is a closed preorder on $(X, t)$ and since $A$ and $B$ respectively are closed subsets of $(X, t)$ it follows from the definition of $A_{r}$ and $B_{l}$ respectively that $A \times A_{r}$ and $B_{l} \times B$ respectively are closed subsets of $X \times X$ endowed with the product topology $t \times t$ on $X \times X$. Hence, $\precsim^{t}$ is the uniquely determined smallest closed preorder on $(X, t)$ that includes $\precsim$ and has the additional property that $A \times A \cup B \times B$ is contained in $\precsim t$. Assertion (i) implies that $\precsim^{t}$ satisfies cmp. Since $a \sim^{t} \tilde{a}$ for all $a \in A$ and all $\tilde{a} \in A$ and $b \sim^{t} \tilde{b}$ for all $b \in B$ and all $\tilde{b} \in B$ we, thus, may conclude that there exists some increasing function $f_{A B}:\left(X, \precsim^{t}, t\right) \longrightarrow\left([0,1], \leq, t_{\text {nat }}\right)$ such that $f_{A B}(A)=\{0\}$ and $f_{A B}(B)=\{1\}$. The inclusion $\precsim \subset \precsim^{t}$ then implies that $f_{A B}$ also is increasing with respect to $\precsim$. An application of Nachbin's Separation Theorem [17, Theorem 1], therefore finishes the proof of the implication.

(ii) $\Rightarrow$ (i): Let $\precsim$ be a closed preorder on $(X, t)$ and let two points $x \in X$ and $y \in X$ such that $\operatorname{not}(y \precsim x)$ be arbitrarily chosen. Since $\precsim$ is closed it follows, in particular, that $\precsim$ is semi-closed. Hence the sets $d(x)$ and $i(y)$ are disjoint closed decreasing, respectively increasing subsets of $X$. The normality of $\precsim$ implies with help of the well known Separation Theorem of Nachbin [17, Theorem 1] that there exists a continuous and increasing function $f_{x y}:(X, \precsim, t) \longrightarrow\left([0,1], \leq, t_{n a t}\right)$ such that $f_{x y}(x)=0$ and $f_{x y}(y)=1$. Lemma 1.1 , therefore, implies the validity of assertion (i).

Theorem 3.4 provides a first step towards the complete solution of the problem of characterizing all topological spaces $(X, t)$ for which every closed preorder $\precsim$ on $(X, t)$ satisfies cmp. It proves that for Hausdorff spaces this problem is equivalent to the problem of characterizing all topological spaces that have the property that every closed preorder $\precsim$ on $(X, t)$ is normal. In contrast, Lemma 3.2 implies that a strongly normal preorder $\precsim$ on $(X, t)$ is always $D$ - $I$-closed. Hence, we may conclude with help of Proposition 3.3 that in order that on $\left(\mathbb{R}, t_{n a t}\right)$ every closed preorder is strongly normal it is necessary that $\left(\mathbb{R}, t_{\text {nat }}\right)$ is sequentially compact. This contradiction implies that even on $\left(\mathbb{R}, t_{\text {nat }}\right)$ there exist closed preorders that are not strongly normal. This means that Theorem 3.4 cannot be improved by replacing "normal" by "strongly normal". 
We now come to the main results of this paper. Indeed, the following Theorem 3.5 may be interpreted as being the converse of Levin's theorem. Since a locally and $\sigma$-compact Hausdorff-space is paracompact the intimate connection of Theorem 3.5 to Levin's theorem is obvious. Its only lack, therefore, is the additional assumption $(X, t)$ to be first countable. But at present the authors do not see any possibility of how to really avoid this assumption in a satisfactory way.

In order to prove Theorem 3.5 we still must verify the validity of the following proposition.

Proposition 3.5 Let $(X, t)$ be a Hausdorff-space and let $\left(X \backslash S, t_{\mid X \backslash S}\right)$ be compact. Then every closed preorder $\precsim$ on $(X, t)$ is normal.

Proof: Let $\precsim$ be some closed preorder on $(X, t)$. In order to prove that $(X, \precsim, t)$ is a normally preordered space, let $A$ and $B$ be two disjoint closed decreasing respectively, increasing subsets of $X$. We must show that there exists disjoint open decreasing respectively, increasing subsets $U$ and $V$ of $X$ such that $A \subset U$ and $B \subset V$. In order to prove the existence of $U$ and $V$ respectively we distinguish between the case that at least one of the sets $A$ and $B$ is a subset of $S$ and the case that neither $A$ nor $B$ is a subset of $S$. Because of the properties of $S$ the first case does not need any reflection. Therefore we now may concentrate on the situation that neither $A \cap(X \backslash S)$ nor $B \cap(X \backslash S)$ is empty. Since $\precsim$ is a closed preorder on $(X, t)$ and since $\left(X \backslash S, t_{\mid X \backslash S}\right)$ is a compact Hausdorff space we may use, in particular, a straightforward modification of the proof of Theorem 4 in Chapter 3 on compact ordered spaces in Nachbin [17] in order to conclude that disjoint open subsets $O_{0}$ and $P_{0}$ of $X$ that contain $A$ and $B$ respectively can be chosen in such a way that $O_{0} \cap(X \backslash S)$ is decreasing and $P_{0} \cap(X \backslash S)$ is increasing with respect to $\precsim_{\mid X \backslash S}$. Now we proceed by following the spirit of the implication "ST $\Rightarrow$ SM" of the proof of Theorem 3.2. Using the assumption $\precsim$ to be a closed preorder on $(X, t)$ this means that the same arguments that have been applied in the proof of the implication "ST $\Rightarrow \mathbf{S M}$ " of the proof of Theorem 3.2 now allow us to assume without loss of generality that $d\left(\overline{d\left(O_{0}\right)}\right) \cap i\left(\overline{i\left(P_{0}\right)}\right)=\emptyset$. Hence, $\overline{d\left(O_{0}\right)}$ and $\overline{i\left(P_{0}\right)}$ are disjoint closed subsets of $X$ such that not $(y \precsim x)$ for all points $x \in \overline{d\left(O_{0}\right)}$ and all points $y \in \overline{i\left(P_{0}\right)}$. This conclusion now allows us to continue the proof of Proposition 3.5 by using $\overline{d\left(O_{0}\right)}$ and $\overline{i\left(P_{0}\right)}$ respectively instead of $A$ and $B$ respectively. This means that we now continue by constructing open subsets $O_{1}$ and $P_{1}$ of $X$ that contain $\overline{d\left(O_{0}\right)}$ and $\overline{i\left(P_{0}\right)}$ respectively such that $d\left(\overline{d\left(O_{1}\right)}\right) \cap i\left(\overline{i\left(P_{1}\right)}\right)=\emptyset$. Continuing inductively in this way we, thus, obtain for every $n \in \mathbb{N}$ open subsets $O_{n}$ and $P_{n}$ of $X$ such that $A \subset O_{n} \subset \overline{d\left(O_{n}\right)} \subset O_{n+1}$, $B \subset P_{n} \subset \overline{i\left(P_{n}\right)} \subset P_{n+1}$ and $d\left(\overline{d\left(O_{n+1}\right)}\right) \cap i\left(\overline{i\left(P_{n+1}\right)}\right)=\emptyset$. As in the proof of the implication "ST $\Rightarrow \mathbf{S M}$ " of the proof of Theorem 3.2 we, therefore, may finally conclude that $U:=\bigcup_{n \in \mathbb{N}} O_{n}$ and $V:=\bigcup_{n \in \mathbb{N}} P_{n}$ are disjoint open decreasing respectively, increasing open subsets of $X$ that contain $A$ and $B$ respectively. 
This conclusion completes the proof of the proposition.

In order to successfully continue we still need the following immediate corollary that is an immediate consequence of Proposition 3.5 and the proof of the implication "(ii) $\Rightarrow$ (i)" of Theorem 3.4.

Corollary 3.1 Let $(X, t)$ be a Hausdorff-space and let $\left(X \backslash S, t_{\mid X \backslash S}\right)$ be compact. Then every closed preorder $\precsim$ on $(X, t)$ satisfies cmp.

Now we are fully prepared for proving the main theorem of this paper.

Theorem 3.5 Let $(X, t)$ be a first countable paracompact space. Then $\left(X \backslash S, t_{\mid X \backslash S}\right)$ is compact and every closed preorder $\precsim$ on $(X, t)$ satisfies cmp or $\left(X \backslash S, t_{\mid X \backslash S}\right)$ is not compact and the assumption that every closed preorder $\precsim$ on $(X, t)$ satisfies cmp implies that $(X, t)$ is the direct sum of locally and $\sigma$-compact Hausdorff spaces.

Proof: Since a paracompact space is normal our assumption on $(X, t)$ to be a Fréchet space implies that $(X, t)$ is a Hausdorff space (cf. the above proof of Proposition 3.4). The proof of Theorem 3.5, therefore, is based upon Theorem 3.3 and the following theorem that is well known in general topology (cf., for instance, Grotemeyer [8, Satz 97]).

Theorem 3.6 Let $(X, t)$ be a locally compact topological space. Then the following assertions are equivalent:

(i) $(X, t)$ is paracompact..

(ii) $(X, t)$ is the direct sum of locally and $\sigma$-compact topological spaces.

We continue by arbitrarily choosing some in the remainder of the proof fixed given first countable paracompact space $(X, t)$. Because of Corollary 3.1 we may assume without loss of generality that $\left(X \backslash S, t_{\mid X \backslash S}\right)$ is not compact and that $(X, t)$ has the property that every closed preorder $\precsim$ on $(X, t)$ satisfies cmp. Because of Theorem 3.6 it suffices to prove that $(X, t)$ is locally compact. Let us assume in contrast that $(X, t)$ is not locally compact. Then there exists some point $z \in X \backslash S$ that does not have a compact neighbourhood. Since $\left(X \backslash S, t_{\mid X \backslash S}\right)$ is not compact we now apply Theorem 3.3 in order to conclude that there exists some sequence $\left(x_{n}\right)_{n \in \mathbb{N}}$ of points $x_{n} \in X \backslash S$ that does not have a limit point. This means that we may choose in particular some closed neighborhood $C(z)$ of $z$ and some open neighborhood $O(z)$ of $z$ such that the following conditions hold.

LP1: $O(z) \subset C(z)$.

LP2: $x_{n} \in C(z) \backslash O(z)$ for every $n \in \mathbb{N}$. 
Let $C$ be the closed subset of $X$ that consists of all points $x_{n}$ where $n$ runs through $\mathbb{N}$. Then we proceed by choosing for every $n \in \mathbb{N}$ some sequence $\left(x_{n k}\right)_{k \in \mathbb{N}}$ of points $x_{n k} \in X$ such that $\lim _{k \rightarrow \infty} x_{n k}=x_{n}$ for all $n \in \mathbb{N}$. Condition LP2 allows us to assume, in addition, that there exists some point $q \in C(z) \backslash(O(z) \cup C)$ that is different from all points $x_{n}$ and $x_{n k}$ respectively. Since there exists no closed neighborhood $C^{\prime}(z)$ of $z$ for which every sequence $\left(z_{n}\right)_{n \in \mathbb{N}}$ of points $z_{n} \in C^{\prime}(z)$ has a limit point we now may construct inductively families $\left\{C_{n}(z)\right\}_{n \in \mathbb{N}}$ and $\left\{O_{n}(z)\right\}_{n \in \mathbb{N}}$ of closed neighborhoods of $z$ and open neighbourhoods of $z$ respectively in such a way that the following conditions are satisfied.

L1: $C_{0}(z) \subset O(z)$.

L2: $O_{n+1}(z) \subset C_{n+1}(z) \subset O_{n}(z)$ for all $n \in \mathbb{N}$.

L3: For every $n \in \mathbb{N}$ there exists some sequence $\left(y_{n k}\right)_{k \in \mathbb{N}}$ of points $y_{n k} \in C_{n}(z) \backslash O_{n}(z)$ that has no limit point.

L4: $\bigcap_{n \in \mathbb{N}} O_{n}(z)=\{z\}$.

Now we define a binary relation $\precsim$ on $(X, t)$ by setting

$\precsim:=\Delta_{X} \cup\left\{\left(x_{n k}, y_{n k}\right) \mid(n, k) \in \mathbb{N} \times \mathbb{N}\right\} \cup\left\{\left(q, x_{n}\right) \mid n \in \mathbb{N}\right\}$.

Since there do not exist any three points $u \in X, v \in X$ and $w \in X$ such that $u \prec v$ and $v \prec w$ the definition of $\precsim$ allows us conclude that $\precsim$ is a (pre)order on $X$. Since, in addition, none of the sequences $\left(x_{n}\right)_{n \in \mathbb{N}}$ and $\left(y_{n k}\right)_{k \in \mathbb{N}}$ has a limit point and since $\left(q, x_{n}\right) \in \precsim$ for all $n \in \mathbb{N}$ it follows that $\precsim$, actually, is a closed subset of $X \times X$. Hence, $\precsim$ is a closed (pre)order on $(X, t)$. Furthermore, the definition of $\precsim$ implies that $\operatorname{not}(q \precsim z)$. In order to, therefore, finish the theorem it suffices to show that there exists no continuous and increasing function $f_{z q}:(X, \precsim, t) \longrightarrow\left(\mathbb{R}, \leq, t_{n a t}\right)$ such that $f_{z q}(z)<f_{z q}(q)$. Let us assume, in contrast, that there exists some continuous and increasing function $f_{z q}:(X, \precsim, t) \longrightarrow\left(\mathbb{R}, \leq, t_{n a t}\right)$ such that $f_{z q}(z)<f_{z q}(q)$. Then we arbitrarily choose some fixed real number $\eta$ such that $f_{z q}(z)<\eta<f_{z q}(q)$. The strict relation $x_{n k} \prec y_{n k}$ for all $n \in \mathbb{N}$ and $k \in \mathbb{N}$ implies that $f_{z q}\left(x_{n k}\right) \leq$ $f_{z q}\left(y_{n k}\right)$ for all $n \in \mathbb{N}$ and all $k \in \mathbb{N}$. But since for all $k \in \mathbb{N}$ the sequences $\left(y_{n k}\right)_{n \in \mathbb{N}}$ uniformly converge to $z$ there exists some $N \in \mathbb{N}$ such that $f_{z q}\left(y_{N k}\right)<$ $\eta$ for all $k \in \mathbb{N}$. Therefore, the equation $\lim _{k \rightarrow \infty} x_{N k}=x_{N}$ allows us to conclude with help of the definition of $\precsim$ that $f_{z q}\left(x_{N}\right)=\lim _{k \rightarrow \infty} f_{z q}\left(x_{N k}\right) \leq \lim _{k \rightarrow \infty} f_{z q}\left(y_{N k}\right) \leq$ $\eta<f_{z q}(q)$. Conversely, the inequality $q \prec x_{N}$ implies that $f_{z q}(q) \leq f_{z q}\left(x_{N}\right)$. Hence, $f_{z q}$ cannot be increasing. This contradiction completes the proof of the second case and, therefore, of the theorem. 
A thorough analysis of the proof of Theorem 3.5 allows us to state the following most general theorem that can be proved with help of the methods that have been developed in this paper.

Theorem 3.7 Let $(X, t)$ be a Hausdorff space. Then $\left(X \backslash S, t_{\mid X \backslash S}\right)$ is compact and every closed preorder $\precsim$ on $(X, t)$ satisfies cmp or $\left(X \backslash S, t_{\mid X \backslash S}\right)$ is not compact and the assumptions that $(X, t)$ is first countable and that every closed preorder $\precsim$ on $(X, t)$ satisfies cmp imply that $(X, t)$ is locally sequentially compact.

Example 3.1 Of course, there exist complete second countable metric spaces $\left(X, t_{d}\right)$ that are not locally compact but have the property that $\left(X \backslash S, t_{d \mid X \backslash S}\right)$ is compact. Indeed, let $\mathbb{N}^{*}:=\mathbb{N} \backslash\{0\}$ and $X:=\{0\} \cup \mathbb{N}^{*} \times \mathbb{N}^{*}$ endowed with the metric $d: X \times X \longrightarrow \mathbb{R}^{\geq 0}$ that is defined by setting

$$
d(x, y):=d(y, x):= \begin{cases}0 & \text { if } x=y \\ \frac{1}{n} & \text { if } x=0 \text { and } y=(n, k) \text { for some pair } \\ & (n, k) \in \mathbb{N}^{*} \times \mathbb{N}^{*} \\ \frac{1}{n}+\frac{1}{m} & \text { if } x=(n, k) \in \mathbb{N}^{*} \times \mathbb{N}^{*} \text { and } \\ y=(m, t) \in\left(\mathbb{N}^{*} \times \mathbb{N}^{*}\right) \backslash\{(n, k)\}\end{cases}
$$

for all pairs $(x, y) \in X \times X$. Then $\left(X, t_{d}\right)$ has the desired properties.

In order to complete Example 3.1 we mention that

$$
(X, d):=\left(\left\{\frac{1}{n} \mid n \in N^{*}\right\},|\cdot|\right)
$$

is a metric space that is not complete but, nevertheless, has the property that all its closed preorders satisfy cmp.

In addition, we still mention that the rationals $\mathbb{Q}$ endowed with its natural metric $d:=|\cdot|$ neither have the property that $\left(\mathbb{Q} \backslash S, t_{d \mid \mathbb{Q} \backslash S}\right)$ is compact nor the property that $\left(\mathbb{Q}, t_{d}\right)$ is locally compact. Therefore, $\left(\mathbb{Q}, t_{d}\right)$ is a second countable metric space for which not every closed preorder that is definable on $\left(\mathbb{Q}, t_{d}\right)$ satisfies cmp.

Since metrizable spaces are paracompact the first result on metrizable spaces that has been mentioned in the introduction is an immediate consequence of Theorem 3.5.

In addition, with respect to Levin's theorem the following first corollary of Theorem 3.5 is of particular interest. 
Corollary 3.2 Let $(X, t)$ be a first countable Lindelöf space. Then the following assertions are equivalent:

(i) Every closed preorder $\precsim$ on $(X, t)$ satisfies cmp.

(ii) $\left(X \backslash S, t_{\mid X \backslash S}\right)$ is a compact Hausdorff-space and $S$ is a countable set or $(X, t)$ is a locally and $\sigma$-compact Hausdorff space.

\section{Proof:}

(i) $\Rightarrow$ (ii). Assertion (i) implies with help of Theorem 3.4 that $(X, t)$ is a normal space. Since every regular Lindelöf space is paracompact Proposition 3.4 and Theorem 3.5, therefore, imply with help of the Lindelöf property of $(X, t)$ that $\left(X \backslash S, t_{\mid X \backslash S}\right)$ is a compact Hausdorff-space and $S$ is a countable set or that $(X, t)$ is the direct sum of countably many locally and $\sigma$-compact Hausdorff spaces, which means that $(X, t)$ is a locally and $\sigma$-compact Hausdorff space.

(ii) $\Rightarrow$ (i). This implication is a consequence of Proposition 3.4 and Levin's theorem respectively.

Since second countable spaces are Lindelöf and since second countable paracompact Hausdorff spaces are metrizable and since, in addition, compact metrizable spaces are second countable the second result on metrizable spaces that has been mentioned in the introduction is an immediate consequence of Corollary 3.2.

If $(X, t)$ is connected then $S$ is empty. Hence, the third result on metrizable spaces that has been mentioned in the introduction follows from the following corollary of Theorem 3.5.

Corollary 3.3 Let $(X, t)$ be a first countable connected paracompact space. Then the following assertions are equivalent:

(i) Every closed preorder $\precsim$ on $(X, t)$ satisfies cmp..

(ii) $(X, t)$ is a locally and $\sigma$-compact Hausdorff space.

It is well known that a Hausdorff topological vector space $(X,+, t)$ is finite-dimensional if and only if it is locally compact. Hence, the following result that generalizes Example 1 in Evren and Ok [6] also is a corollary of Theorem 3.5.

Corollary 3.4 Let $(X,\|\cdot\|)$ be a normed linear space. Then the following assertions are equivalent: 
(i) Every closed preorder $\precsim$ on $(X,\|\cdot\|)$ satisfies cmp..

(ii) $X$ is finite-dimensional.

Banach spaces $(X,\|\cdot\|)$ that are not finite dimensional, therefore, are complete metric spaces that have the property that not every closed preorder that is definable on $(X,\|\cdot\|)$ satisfies cmp. The Banach space of all continuous real-valued functions on some compact non-degenerate real interval, thus, is a complete second countable metric space that has the property that not all closed preorders that are definable on this space satisfy cmp (a further example of this type is Example 1 in Evren and Ok [6]).

In the remainder of this section we want to discuss the problem if the necessary condition the validity of which has been proved in Theorem 3.5 also is sufficient in order to guarantee that every closed preorder $\precsim$ on $(X, t)$ satisfies cmp. In order to be more precise, let $(X, t)$ be the direct sum of locally and $\sigma$-compact Hausdorff spaces. Then we want to discuss the question if these assumptions imply that every closed preorder $\precsim$ on $(X, t)$ satisfies cmp? Therefore, we somewhat modify Theorem 3.5 by requiring, in addition, $(X, t)$ to be locally connected. Then the following corollary of Theorem 3.5 holds.

Corollary 3.5 Let $(X, t)$ be a first countable paracompact and locally connected space. Then $\left(X \backslash S, t_{\mid X \backslash S}\right)$ is compact and every closed preorder $\precsim$ on $(X, t)$ satisfies cmp or $\left(X \backslash S, t_{\mid X \backslash S}\right)$ is not compact and the assumption that every closed preorder $\precsim$ on $(X, t)$ satisfies cmp implies that $(X, t)$ is the direct sum of connected locally and $\sigma$-compact Hausdorff spaces.

On basis of this corollary we are ready for proving the following proposition that provides a first answer of the question that has been posed above.

Proposition 3.6 Let $(X, t)$ be the direct sum of connected locally and $\sigma$-compact Hausdorff spaces. Then every closed preorder $\precsim$ on $(X, t)$ admits a continuous multi-utility representation.

Proof: Let $x \in X$ be arbitrarily chosen. Then we denote, for the moment, by $C(x)$ the component of $X$ that contains $x$. With help of this notation we are able to prove the following lemma that is essential for the proof of the proposition.

Lemma 3.3 Let $\precsim$ be a closed preorder on $(X, t)$ the indifference classes $[q]$ of which are contained in $C(q)$. Then for any three points $x \in X, y \in X$ and $z \in X$ for which the equation $C(x)=C(z)$ and the inequalities $x \prec y \prec z$ hold the equations $C(x)=C(y)=C(z)$ are satisfied. 
Proof: Let us assume, in contrast, that $y \notin C(x)=C(z)$. Then we may conclude with help of the assumptions of Lemma 3.4 that $A:=d(y) \cap C(x)=$ $d(y) \cap C(z)$ and $B:=i(y) \cap C(x)=i(y) \cap C(z)$ are disjoint closed decreasing respectively, increasing subsets of $X$. Therefore, we set $C:=C(x) \cup[y]=$ $C(z) \cup[y]$ in order to consider the closed preorder $\precsim_{\mid C}$ on $\left(C, t_{\mid C}\right)$. Levin's theorem now implies that $\precsim_{\mid C}$ has a continuous multi-utility representation. Hence, considering the pairs $(x, y) \in \prec_{\mid C}$ and $(y, z) \in \prec_{\mid C}$ we obtain two appropriate increasing functions the sum of which guarantees the existence of some continuous and increasing function $f:\left(C, \precsim_{\mid C}, t_{\mid C}\right) \longrightarrow\left(\mathbb{R}, \leq, t_{\text {nat }}\right)$ such that $f(u)<f(y)<f(v)$ for all points $u \in C(x)=C(z)$ and $v \in C(x)=C(z)$ such that $u \prec y \prec v$. We proceed by setting $D:=\{u \in C(x)=C(z)$ $f(u) \leq f(y)\}$. Then it follows that $D$ and $B$ are disjoint non-empty closed subsets of $C(x)=C(z)$ the union of which is $C(x)=C(z)$. Since $C(x)=C(z)$ is an open subset of $X$ this last conclusion contradicts the connectedness of $C(x)=C(z)$.

Let $\precsim$ be some fixed given closed preorder on $(X, t)$. In order to now prove Proposition 3.6 we must show that $\precsim$ satisfies cmp. Therefore, we choose in a first step in every indifference class of $\precsim$ some fixed point $q$ in order to then denote the collection of these points by $F$. Now we replace $\precsim$ by

$\precsim^{\prime}:=\precsim \backslash\{(r, s) \in X \times X \mid$ there exists some $q \in F$ such that $[r]=[s]=[q]$ and $r \notin C(q)$ or $s \notin C(q)\}$.

The definition of $\precsim^{\prime}$ implies that $\precsim^{\prime}$ is a closed preorder on $(X, t)$. Furthermore, Lemma 1.1 allows us to conclude that $\precsim$ satisfies cmp if and only if $\precsim^{\prime}$ satisfies cmp. Hence, it suffices to verify that $\precsim^{\prime}$ satisfies cmp. Let, therefore, $x \in X$ and $y \in X$ such that $\operatorname{not}(y \precsim x)$ be arbitrarily chosen. Then either the equality $C(x)=C(y)$ or the inequality $C(x) \neq C(y)$ is possible. Since the case that $C(x)=C(y)$ is somewhat more complicated than the case that $C(x) \neq C(y)$ and since, in addition, both cases can be settled by analogous arguments, in the remainder of the proof of Proposition 3.6, we merely concentrate on the equation $C(x)=C(y)$. We, thus, set $C:=C(x)=C(y)$ in order to then conclude that Levin's theorem guarantees the existence of some continuous increasing function $f:\left(C, \precsim_{\mid C}^{\prime}, t_{\mid C}\right) \longrightarrow\left(\mathbb{R}, \leq, t_{\text {nat }}\right)$ such that $f(x)<f(y)$. In order to now finish the proof of the proposition it suffices to show because of Lemma 1.1 that $f$ can be lifted to some continuous and increasing function $h:\left(X, \precsim^{\prime}, t\right) \longrightarrow\left(\mathbb{R}, \leq, t_{\text {nat }}\right)$. Let, therefore, some point $z \in X \backslash C$ be arbitrarily chosen. Then we have to distinguish between the following three cases.

Case 1: $z \prec^{\prime} x$. In this case we set $h(v):=f(x)$ for all $v \in C(z)$.

Case 2: $x \prec^{\prime} z$. Now we set $h(v):=f(y)$ for all $v \in C(z)$. 
Case 3: There exists neither a point $u \in C(z)$ such that $u \prec^{\prime} x$ nor a point $w \in C(z)$ such that $x \prec^{\prime} w$. In this situation we set $h(v):=f(x)$ for all $v \in C(z)$.

Setting $h_{\mid C}=f$ in this way a lifting of $f$ has been defined. In addition, Lemma 3.4 allows us to conclude that $h:\left(X, \precsim^{\prime}, t\right) \longrightarrow\left(\mathbb{R}, \leq, t_{\text {nat }}\right)$ is continuous and increasing and satisfies the inequality $h(x)<h(y)$. This conclusion finishes the proof of Proposition 3.6.

With help of Proposition 3.6 the proof of Theorem 3.5 implies the following theorem which, in addition, at least partly answers the above question and, therefore, provides a good opportunity of finishing this section.

Theorem 3.8 Let $(X, t)$ be a first countable paracompact and locally connected space. Then the following assertions are equivalent:

(i) Every closed preorder $\precsim$ on $(X, t)$ satisfies cmp.

(ii) $\left(X \backslash S, t_{\mid X \backslash S}\right)$ is compact or $(X, t)$ is the direct sum of connected locally and $\sigma$-compact Hausdorff spaces.

\section{Conclusion}

Although the problem of characterizing all topological spaces for which every closed preorder admits a continuous multi-utility representation could not completely be solved by the authors the corresponding results of this paper are very restrictive. Indeed, they imply, for example, that spaces like $L^{\infty}(\mu)$, the space of essentially bounded measurable functions relative to a $\sigma$-finite measure $\mu$, always allow the definition of closed preorders that do not have a continuous multi-utility representation. On the other hand topological vector spaces such as $L^{\infty}(\mu)$ meanwhile often are used as infinite-dimensional commodity spaces in economic theory. Therefore, it is desirable to have continuous multi-representation theorems that apply to them. This means that one has to replace the particular linearly ordered abelian group $(\mathbb{R},+, \leq)$ by an arbitrary linearly ordered abelian group $(\mathbb{A},+, \leq)$. Such a more general approach meanwhile has been started by Pivato [19].

In addition, in Herden and Mehta [11] it has been underlined by many examples that the real line often is not the appropriate codomain of a utility function and that, consequently, it is worthwhile to develop a theory of continuous non-real valued utility functions. These considerations also underline that particular value of an approach as presented by Pivato [19].

Nevertheless, the problem of characterizing all topological spaces for which every closed preorder allows a continuous multi-utility representation is still 
pressing. In this sense Theorem 3.4, Theorem 3.5, Theorem 3.7 and Theorem 3.8 provide first results that at least prepare a complete solution of this problem.

\section{References}

[1] Alcantud, J.C.R., Bosi, G., Zuanon, M., Richter-Peleg multi-utility representations of preorders, submitted.

[2] Bosi, G., Herden, G., Continuous multi-utility representations of preorders, Journal of Mathematical Economics 48 (2012), 212-218.

[3] Bosi, G., Zuanon, M., Continuous multi-utility for extremely continuous preorders, International Journal of Contemporary Mathematical Sciences 4 (2009), $439-445$

[4] Burgess, D.C.J., Fitzpatrick, M., On separation axioms for certain types of ordered topological space, Mathematical Proceedings of the Cambridge Philosophical Society 82 (1977), 59-65.

[5] Evren, O., On the existence of expected multi-utility representations, Economic Theory 35 (2008), 575-592.

[6] Evren, O., Ok, E.A., On the multi-utility representation of preference relations, Journal of Mathematical Economics 47 (2011), 554-563.

[7] Galaabaatar, T., Karni, E., Expected multi-utility representations, Mathematical Social Sciences 64 (2012), 242-246.

[8] Grotemeyer, K.P., Topologie, Mathematisches Institut der Freien Universität, 1966.

[9] Herden, G., On the existence of utility functions, Mathematical Social Sciences 17 (1989), 297-313.

[10] Herden, G., On a lifting theorem of Nachbin, Mathematical Social Sciences 19 (1990), 37-44.

[11] Herden, G., Mehta, G.B., The Debreu Gap Lemma and some generalizations, Journal of Mathematical Economics 40 (2004), 747-769.

[12] Levin, V.L., A continuous utility theorem for closed preorders on a $\sigma$-compact metrizable space, Soviet Mathematics Doklady 28 (1983), 715718.

[13] Levin, V.L., Measurable utility theorem for closed and lexicographic preference relations, Soviet Mathematics Doklady 27 (1983), 639643.

[14] Mas-Colell, A., Whinston, M.D., Green, J.R., Microeconomic Theory, Oxford University Press, 1995. 
[15] Minguzzi, E., Topological conditions for the representation of preorders by continuous utilities, Applied General Topology 13 (2012), 81-89.

[16] Minguzzi, E., Normally Preordered Spaces and Utilities, Order 30 (2013),137150.

[17] Nachbin, L.: Topology and order, Van Nostrand, Princeton, 1965.

[18] Nosratabadi, H.: Partially upper continuous preferences: Representation and maximal elements, Economics Letters 125 (2014), 408410.

[19] Pivato, M., Multiutility representations for incomplete difference preorders, Mathematical Social Sciences 66 (2013), 196-220. 